\title{
Practical Stability in terms of Two Measures for Set Differential Equations on Time Scales
}

\author{
Peiguang Wang ${ }^{1}$ and Weiwei Sun ${ }^{2}$ \\ ${ }^{1}$ College of Electronic and Information Engineering, Hebei University, Baoding 071002, China \\ ${ }^{2}$ College of Mathematics and Computer Science, Hebei University, Baoding 071002, China \\ Correspondence should be addressed to Peiguang Wang; pgwang@mail.hbu.edu.cn
}

Received 8 November 2013; Accepted 24 December 2013; Published 20 January 2014

Academic Editors: N. Henderson and Y. Wu

Copyright (c) 2014 P. Wang and W. Sun. This is an open access article distributed under the Creative Commons Attribution License, which permits unrestricted use, distribution, and reproduction in any medium, provided the original work is properly cited.

\begin{abstract}
We present a new comparison principle by introducing a notion of upper quasi-monotone nondecreasing and obtain the practical stability criteria for set valued differential equations in terms of two measures on time scales by using the vector Lyapunov function together with the new comparison principle.
\end{abstract}

\section{Introduction}

Stability theory in the sense of Lyapunov is now well known. Its basic theory and applications can be found in the monographs of Lasalle and Lefschetz [1] and Rouche et al. [2]. To unify a variety of stability concepts and to offer a general framework for the investigation of the stability theory, the notion of stability in terms of two measures has been proved to be very powerful (see the monograph [3] and the papers [4-6]).

The practical stability is a very important problem in the field of application, which deals with the question of whether the system state evolves within certain subsets of the statespace. It is very useful in estimating the worst-case transient and steady-state responses and in verifying pointwise in time constraints imposed on the state trajectories. Thus practical stability is concerned with quantitative analysis as opposed to Lyapunov analysis which is qualitative in nature. There are some relative results for practical stability of various dynamic systems. We can refer to the monograph of Lakshmikantham et al. [7] and the papers of Zhang and Sun [8], Wang and Liu [9], Wang et al. [10], Sun et al. [11], and Hristova and Georgieva [12] and the references cited therein.

Recently, the study of set differential equations in a semilinear metric space has gained much attention due to its applicability to multivalued differential inclusions and fuzzy differential equations and its inclusion of ordinary differential systems as a special case [13], and some basic results of interest are obtained in [14-21]. However, we notice that there are very few results for set valued differential equation on time scales. For example, Girton [22] gave the results of the existence and uniqueness of the solution of an initial value problem that involve set valued differential equations on time scales. Ahmad and Sivasundaram [23] and Hong [24, 25] discussed some basic problems of set valued differential equation on time scales and obtained some stability criteria, respectively. In this paper, we present a new comparison principle by introducing a notion of upper quasi-monotone nondecreasing and obtain the practical stability criteria for set valued differential equations in terms of two measures on time scales by using the vector Lyapunov function together with the new comparison principle. Consequently, this paper is organized as follows. In Section 2, we introduce the concepts of the time scales and the set valued differential equations, the propositions of the set valued differential equations on time scales. In Section 3, the relatively new comparison principle and conditions of stability are given.

\section{Preliminaries}

Let $\mathbb{T}$ be a time scale with $t_{0} \geq 0$ as minimal element and no maximal element. Firstly, we give some relative definitions, which can be found in [1-3]. 
Definition 1. The mappings $\sigma, \rho: \mathbb{T} \rightarrow \mathbb{T}$ defined as

$$
\sigma(t)=\inf (s \in \mathbb{T} ; s>t), \quad \rho(t)=\sup (s \in \mathbb{T} ; s<t)
$$

are called jump operators.

Definition 2. A nonmaximal element $t \in \mathbb{T}$ is said to be rightscattered (rs) if $\sigma(t)>t$ and right-dense (rd) if $\sigma(t)=t$. A nonminimal element $t \in \mathbb{T}$ is called left-scattered (ls) if $\rho(t)<$ $t$ and left-dense (ld) if $\rho(t)=t$.

Definition 3. Let

$$
\mathbb{T}^{k}= \begin{cases}\mathbb{T} \backslash(\rho(\sup \mathbb{T}), \sup \mathbb{T}) & \text { if } \sup \mathbb{T}<\infty, \\ \mathbb{T} & \text { if } \sup \mathbb{T}=\infty .\end{cases}
$$

Definition 4. The mapping $g$ is called regulated, if in each leftdense $t \in \mathbb{T}$ the left sided and in each right-dense $t \in \mathbb{T}$ the right sided limit exist.

Definition 5. The mapping $g: \mathbb{T} \rightarrow \mathbb{R}^{n}$ is called rd continuous if

(i) it is continuous at each right-dense $t \in \mathbb{T}$;

(ii) at each left-dense point the left-sided limit $g\left(t^{-}\right)$ exists.

Let $C_{\mathrm{rd}}\left[\mathbb{T}, \mathbb{R}^{n}\right]$ denote the set of rd-continuous mappings from $\mathbb{T}$ to $\mathbb{R}^{n}$.

Definition 6. The mapping $f: \mathbb{T} \times \mathbb{R}^{n} \rightarrow \mathbb{R}^{n}$ is said to be right-dense (rd) continuous and is denoted by $f \in C_{\mathrm{rd}}[\mathbb{}[\times$ $\left.\mathbb{R}^{n}, \mathbb{R}^{n}\right]$ if

(i) it is continuous at each $(t, x)$ with right-dense or maximal $t$;

(ii) the limits $f\left(t^{-}, x\right)=\lim _{(s, y) \rightarrow\left(t^{-}, x\right)} f(s, y)$ and $\lim _{y \rightarrow x}$ $f(t, y)$ exist at each $(t, x)$ with left-dense $t$.

Let $K\left(\mathbb{R}^{n}\right)$ denote the collection of all nonempty, compact, and convex subsets of $\mathbb{R}^{n}$. Define the Hausdorff metric

$$
D[A, B]=\max \left[\sup _{x \in B} d(x, A), \sup _{y \in A} d(y, B)\right],
$$

where $d[x, A]=\inf [d(x, y): y \in A], A, B$ are bounded sets in $\mathbb{R}^{n}$. We note that $K\left(\mathbb{R}^{n}\right)$ with this metric is a complete metric space.

It is known that if the space $K\left(\mathbb{R}^{n}\right)$ is equipped with the natural algebraic operations of addition and nonnegative scalar multiplication, then $K\left(\mathbb{R}^{n}\right)$ becomes a semilinear metric space which can be embedded as a complete cone into a corresponding Banach space.

The Hausdorff metric (3) satisfies the following properties:

$$
\begin{gathered}
D[A+C, B+C]=D[A, B], \quad D[A, B]=D[B, A], \\
D[\lambda A, \lambda B]=\lambda D[A, B], \\
D[A, B] \leq D[A, C]+D[C, B],
\end{gathered}
$$

for all $A, B, C \in K\left(\mathbb{R}^{n}\right)$ and $\lambda \in \mathbb{R}_{+}$.
Definition 7. Let $A, B \in K\left(\mathbb{R}^{n}\right)$. The set $C \in K\left(\mathbb{R}^{n}\right)$ satisfying $A=B+C$ is known as the Hukuhara difference of the sets $A$ and $B$ and is denoted by the symbol $A-B$.

Definition 8 . The forward derivative of $F: \mathbb{T} \rightarrow K\left(\mathbb{R}^{n}\right)$, denoted by $\Delta F$, is defined by

$$
\begin{gathered}
\Delta F(t)=\frac{F(\sigma(t))-F(t)}{\sigma(t)-t}, \quad \text { if } t \text { is right-scattered, } \\
\Delta F(t)=\lim _{s \rightarrow t^{+}} \frac{F(s)-F(t)}{s-t}, \quad \text { if } t \text { is right-dense. }
\end{gathered}
$$

Analogously, the backward derivative $\nabla F$ is defined as

$$
\begin{aligned}
& \nabla F(t)=\frac{F(t)-F(\rho(t))}{t-\rho(t)}, \quad \text { if } t \text { is left-scattered, } \\
& \nabla F(t)=\lim _{s \rightarrow t^{-}} \frac{F(t)-F(s)}{t-s}, \quad \text { if } t \text { is left-dense. }
\end{aligned}
$$

Remark 9. The usual scalar subtraction occurs in the scalar coefficient at the beginning of the above expressions and the Hukuhara set difference occurs inside the brackets. Clearly, the existence of Hukuhara difference ensures the existence of the derivative in Definition 8; in the forthcoming analysis, we will be considering the forward derivative as the result for backward derivative followed immediately with suitable changes. The backward derivative has the following properties:

(i) let $F, G: \mathbb{T} \rightarrow K\left(\mathbb{R}^{n}\right)$ be differentiable at $t$; then $\Delta(F+$ $G)(t)=\Delta F(t)+\Delta G(t)$

(ii) $\Delta(\alpha F)(t)=\alpha(\Delta F(t)), \alpha \in \mathbb{R}_{+}$.

Remark 10. If the sets in $K\left(\mathbb{R}^{n}\right)$ are singletons only, then there is only one selector possible, namely, $F$ itself. In this case, the integral reduces to the generalized integral from time scales into $\left(\mathbb{R}^{n}\right)$. The assumption $n=1$ generalizes into the integral for time scales. When $\mathbb{T}$ is everywhere right-dense, then we have $\Delta t=d t$, which results in the conventional formulation for integration of set valued functions. Moreover, for $a, b, c \in$

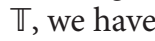

(i) $\int_{a}^{c} F(t) \Delta t=\int_{a}^{b} F(t) \Delta t+\int_{b}^{c} F(t) \Delta t$;

(ii) $\int_{a}^{b} F(t) \Delta t$ is closed and convex but need not be necessarily compact.

Definition 11. Let $F, G: \mathbb{T} \rightarrow K\left(\mathbb{R}^{n}\right)$. Then the Hausdorff distance between $F$ and $G, D[F(\cdot), G(\cdot)]: \mathbb{T} \rightarrow \mathbb{R}_{+}$, is $\Delta$ integrable and

$$
D\left[\int_{t_{0}}^{t} F(s) \Delta s, \int_{t_{0}}^{t} G(s) \Delta s\right] \leq \int_{t_{0}}^{t} D[F(s), G(s)] \Delta s .
$$

Definition 12. Assume $F: \mathbb{T} \rightarrow K\left(\mathbb{R}^{n}\right)$ is set valued function and $t \in \mathbb{T}^{k}$. Let $\Delta_{H} F(t)$ be an element of $K\left(\mathbb{R}^{n}\right)$ (provided it exists) with the property that given any $\epsilon>0$, there exists 
a neighborhood $U_{t}$ of $t$ (i.e., $U_{t}=(t-\delta, t+\delta) \bigcap \mathbb{\mathbb { T }}$ for some $\delta>0)$ such that

$$
\begin{aligned}
& D\left[F(t+h)-f(\sigma(t)), \Delta_{H} F(t)(h-\mu(t))\right] \\
& \quad \leq \epsilon(h-\mu(t)), \\
& D\left[f(\sigma(t))-F(t-h), \Delta_{H} F(t)(\mu(t)+h)\right] \\
& \quad \leq \epsilon(\mu(t)+h)
\end{aligned}
$$

for all $(t-h, t+h) \in U_{t}$ with $0 \leq h<\delta$, where $\mu(t)=$ $\sigma(t)-t$. We call $\Delta_{H} F(t)$ the $\Delta_{H}$-derivative of $F$ at $t$. We say that $F$ is $\Delta_{H}$-differentiable at $t$ if its $\Delta_{H}$-derivative exists at $t$. Moreover, we say $F$ is $\Delta_{H^{-}}$-differentiable on $\mathbb{T}^{k}$ if its $\Delta_{H^{-}}$ derivative exists at each $t \in \mathbb{T}^{k}$. The multivalued function $\Delta_{H} F(t): \mathbb{T}^{k} \rightarrow K\left(\mathbb{R}^{n}\right)$ is then called the $\Delta_{H}$-derivative of $F$ on $T^{k}$.

Proposition 13. Let $F: \mathbb{T} \rightarrow K\left(\mathbb{R}^{n}\right), \Delta_{H} F(t) \in K\left(\mathbb{R}^{n}\right)$ (provided it exists). Then some easy and useful relationships concerning the $\Delta_{H}$-derivative hold.

(i) If the $\Delta_{H^{-}}$derivative of $F$ at $t$ exists, then it is unique. Hence, the $\Delta_{H^{-}}$derivative is well defined.

(ii) Assume $F: \mathbb{T} \rightarrow K\left(\mathbb{R}^{n}\right)$ is a multivalued function and let $t \in \mathbb{T}^{k}$. Then one has the following.

(1) If $F$ is $\Delta_{H}$-differentiable at $t$, then $F$ is continuous at $t$.

(2) If $F$ is continuous at $t$ and $t$ is right-scattered, then $F$ is $\Delta_{H^{-}}$differentiable at $t$ with

$$
\Delta_{H} F(t)=\frac{F(\sigma(t))-F(t)}{\mu(t)} .
$$

(3) Ift is right-dense, then $F$ is $\Delta_{H}$-differentiable at $t$ if the limits

$$
\lim _{h \rightarrow 0^{+}} \frac{F(t+h)-F(t)}{h}, \quad \lim _{h \rightarrow 0^{+}} \frac{F(t)-F(t-h)}{h}
$$

exist and satisfy the equations

$$
\lim _{h \rightarrow 0^{+}} \frac{F(t+h)-F(t)}{h}=\lim _{h \rightarrow 0^{+}} \frac{F(t)-F(t-h)}{h}=\Delta_{H} F(t) .
$$

(4) If $F$ is differentiable at $t$, then

$$
F(\sigma(t))=F(t)+\mu(t) \Delta_{H} F(t) .
$$

Remark 14. Let $F: \mathbb{T} \rightarrow K\left(\mathbb{R}^{n}\right), \Delta_{H} F(t) \in K\left(\mathbb{R}^{n}\right)$ (provided it exists). We consider the two cases $\mathbb{T}=\mathbb{R}$ and $\mathbb{T}=\mathbb{Z}$, where $\mathbb{Z}$ stands for the set consisting of all integers.

(1) If $\mathbb{T}=\mathbb{R}$, then $F: \mathbb{R} \rightarrow K\left(\mathbb{R}^{n}\right)$ is $\Delta_{H^{-}}$-differentiable at $t \in \mathbb{R}$ if and only if

$$
D_{H} F(t)=\lim _{h \rightarrow 0^{+}} \frac{F(t+h)-F(t)}{h}=\lim _{h \rightarrow 0^{+}} \frac{F(t)-F(t-h)}{h}
$$

exists, that is, if and only if $F$ is differentiable in the Hukuhara sense at $t$. In this case, we have

$$
D_{H} F(t)=\Delta_{H} F(t) .
$$

(2) If $\mathbb{T}=\mathbb{Z}$, then $F: \mathbb{Z} \rightarrow K\left(\mathbb{R}^{n}\right)$ is $\Delta_{H}$-differentiable at $t \in \mathbb{Z}$ with

$$
\Delta_{H} F(t)=\frac{F(\sigma(t))-F(t)}{\mu(t)}=F(t+1)-F(t)=\Delta F(t),
$$

where $\Delta$ is the usual forward multivalued difference operator.

\section{Main Results}

Consider the space

$$
\begin{array}{r}
K\left(\mathbb{R}^{n}\right)^{N}=K\left(\mathbb{R}^{n}\right) \times K\left(\mathbb{R}^{n}\right) \times K\left(\mathbb{R}^{n}\right), \ldots, K\left(\mathbb{R}^{n}\right), \\
N \text { times, } N \in Z,
\end{array}
$$

together with either of the following metrics on the space $K\left(\mathbb{R}^{n}\right)^{N}$ :

$$
\widetilde{D}[X, Y]=\left(D\left[X_{1}, Y_{1}\right], D\left[X_{2}, Y_{2}\right], \ldots, D\left[X_{N}, Y_{N}\right]\right),
$$

where $X, Y \in K_{c}\left(R^{n}\right)^{N}, X_{i}, Y_{i} \in K_{c}\left(R^{n}\right), i=1,2, \ldots, N$.

Consider the initial value problem

$$
\Delta_{H} X=F(t, X), \quad X\left(t_{0}\right)=X_{0},
$$

where $F \in C_{\text {rd }}\left[\mathbb{T} \times K\left(\mathbb{R}^{n}\right)^{N}, K\left(\mathbb{R}^{n}\right)^{N}\right], X \in K\left(\mathbb{R}^{n}\right)^{N}, X=$ $\left(X_{1}, X_{2}, \ldots, X_{N}\right)$ such that for each $i, i=1,2, \ldots, N, X_{i} \in$ $K\left(\mathbb{R}^{n}\right)$.

First of all, we define the following classes of function:

$$
\mathscr{K}=\left\{\alpha: \mathbb{R}_{+} \longrightarrow \mathbb{R}_{+},\right.
$$

$\alpha$ is strictly increasing and $\alpha(0)=0\}$,

$\mathscr{C} \mathscr{K}=\left\{\phi: \mathbb{T} \times \mathbb{R}_{+} \longrightarrow \mathbb{R}_{+}, \phi(t, s) \in \mathscr{K}\right.$ for each $\left.t\right\}$,

$$
\begin{gathered}
\Gamma=\left\{h: \mathbb{T} \times K\left(\mathbb{R}^{n}\right)^{N} \longrightarrow \mathbb{R}_{+}, \inf _{X \in K\left(\mathbb{R}^{n}\right)^{N}} h(t, X)=0\right\}, \\
\Sigma=\left\{Q: \mathbb{R}_{+} \longrightarrow \mathbb{R}_{+}, Q(0)=0 \text { and } Q \text { is increasing }\right\}, \\
S(h, \gamma)=\left\{(t, X) \in \mathbb{T} \times K\left(\mathbb{R}^{n}\right)^{N},\right. \\
h \in \Gamma \text { and } h(t, X)<\gamma, \gamma>0\} .
\end{gathered}
$$

In order to discuss the stability of the solution of set valued differential systems (18), we state some notions and definitions. 
On the vector Lyapunov function on time scales, we defined the Dini derivative of the function $V \in C_{\mathrm{rd}}[\mathbb{T} \times$ $K\left(\mathbb{R}^{n}\right)^{N}, \mathbb{R}^{N}$ ] along with the solutions of (18) by

$$
\begin{aligned}
& D_{\Delta}^{-} V(t, X) \\
& =\liminf _{\mu(t) \rightarrow 0^{-}} \frac{V(t, X)-V(t-\mu(t), X(t)-\mu(t) F(t, X))}{\mu(t)}, \\
& D_{\Delta}^{+} V(t, X) \\
& =\limsup _{\mu(t) \rightarrow 0^{+}} \frac{V(t+\mu(t), X(t)+\mu(t) F(t, X))-V(t, X)}{\mu(t)} .
\end{aligned}
$$

Definition 15. A function $G: \mathbb{T} \times \mathbb{R}_{+}^{N} \rightarrow \mathbb{R}^{N}(N \geq 1)$ is said to be upper quasi-monotone nondecreasing in $U$, if $U, W \in \mathbb{R}_{+}^{N}$ and $\|U\|_{M} \leq\|W\|_{M}$ imply $\|G(t, U)\|_{M} \leq\|G(t, W)\|_{M}$, where $\|\cdot\|_{M}=\max _{1 \leq i \leq N} U_{i}$.

In the following, we will prove the comparison result in terms of vector Lyapunov functions relative to the set differential system on time scales.

\section{Theorem 16. Assume that}

$\left(H_{1}\right) V \in C_{\mathrm{rd}}\left[\mathbb{T} \times K\left(\mathbb{R}^{n}\right)^{N}, \mathbb{R}^{N}\right], V$ is locally Lipschitzian in $X$; that is, for $X, Y \in K\left(\mathbb{R}^{n}\right)^{N}$, one has $\mid V(t, X)$ $V(t, Y) \mid \leq A \widetilde{D}[X, Y]$, where $A$ is an $N \times N$ matrix of nonnegative elements, $|V(t, X)-V(t, Y)|=\left(\mid V_{1}(t, X)-\right.$ $V_{1}(t, Y)|,| V_{2}(t, X)-V_{2}(t, Y)|, \ldots,| V_{N}(t, X)-V_{N}$ $(t, Y) \mid)$. Here, by $|V(t, X)|$ one means the vector $\left(\left|V_{1}(t, X)\right|,\left|V_{2}(t, X)\right|, \ldots,\left|V_{N}(t, X)\right|\right)$, where $V_{i}$ are the components of $V, i=1,2, \ldots, N$;

$\left(\mathrm{H}_{2}\right) V$ satisfies

$$
D_{\Delta}^{+} V(t, X) \leq G(t, V(t, X)),
$$

where $G \in C_{\mathrm{rd}}\left[\mathbb{\mathbb { N }} \times \mathbb{R}^{N}, \mathbb{R}^{N}\right]$ is upper quasi-monotone nondecreasing in $U$ for each $t \in \mathbb{T}$;

$$
\left(H_{3}\right) U \in \mathbb{R}^{N}, U(t)=U\left(t, t_{0}, U_{0}\right) \text { is the solution of }
$$

$$
U^{\Delta}=G(t, U), \quad U\left(t_{0}\right)=U_{0} .
$$

Then for any solution $X(t)$ of (18), one has

$$
\|V(t, X)\|_{M} \leq\|U(t)\|_{M}, \quad t \in \mathbb{T},
$$

provided $\left\|V\left(t_{0}, X_{0}\right)\right\|_{M} \leq\left\|U_{0}\right\|_{M}$.

Proof. Let $U(t)$ be the solution of (22) for $t \geq t_{0}, t \in \mathbb{T}$. Set $m(t)=V(t, X(t))$ as an application of the properties of Hausdorff metric; we obtain the estimation

$$
\begin{aligned}
& m(\sigma(t))-m(t) \\
& \quad=V(\sigma(t), X(\sigma(t)))-V(t, X(t))
\end{aligned}
$$

$$
\begin{aligned}
= & V(\sigma(t), X(\sigma(t))) \\
& -V(\sigma(t), X(t)+(\sigma(t)-t) F(t, X(t))) \\
& +V(\sigma(t), X(t)+(\sigma(t)-t) F(t, X(t))) \\
& -V(t, X(t)) \\
\leq & A \widetilde{D}[X(\sigma(t)), X(t)+(\sigma(t)-t) F(t, X(t))] \\
& +V(\sigma(t), X(t)+(\sigma(t)-t) F(t, X(t))) \\
& -V(t, X(t)) .
\end{aligned}
$$

Letting $X(\sigma(t))=X(t)+Y(t)$, where $Y(t)$ is the Hukuhara difference of $X(\sigma(t))$ and $X(t)$ for small $h>0$ and is assumed to exist, hence,

$$
\begin{aligned}
m(\sigma(t)) & -m(t) \\
\leq & A \widetilde{D}[X(t)+Y(t), X(t)+(\sigma(t)-t) F(t, X(t))] \\
& +V(\sigma(t), X(t)+(\sigma(t)-t) F(t, X(t))) \\
& -V(t, X(t)) \\
= & A \widetilde{D}[Y(t),(\sigma(t)-t) F(t, X(t))] \\
& +V(\sigma(t), X(t)+(\sigma(t)-t) F(t, X(t))) \\
& -V(t, X(t)) \\
= & A \widetilde{D}[X(\sigma(t))-X(t),(\sigma(t)-t) F(t, X(t))] \\
& +V(\sigma(t), X(t)+(\sigma(t)-t) F(t, X(t))) \\
& -V(t, X(t)) .
\end{aligned}
$$

Consequently, we find that

$$
\begin{aligned}
& D_{\Delta}^{+} m(t) \\
& =\limsup _{\sigma(t)-t \rightarrow 0^{+}} \frac{m(\sigma(t))-m(t)}{\sigma(t)-t} \\
& =\limsup _{\sigma(t)-t \rightarrow 0^{+}} \frac{1}{\sigma(t)-t} A \widetilde{D}[X(\sigma(t))-X(t), \\
& \quad+\frac{V(\sigma(t), X(t)+(\sigma(t)-t) F(t, X(t)))-V(t, X(t))}{\sigma(t)-t} \\
& \leq A \widetilde{D}\left[\frac{X(\sigma(t))-X(t)}{\sigma(t)-t}, F(t, X(t))\right]+D_{\Delta}^{+} V(t, X)
\end{aligned}
$$




$$
\begin{aligned}
& =A \widetilde{D}\left[\Delta_{H} X(t), F(t, X(t))\right]+D_{\Delta}^{+} V(t, X) \\
& =D_{\Delta}^{+} V(t, X)
\end{aligned}
$$

where $D_{\Delta}^{+} m(t)$ is the right-derivative of $m(t)$. Hence, we have

$$
D_{\Delta}^{+} m(t) \leq G(t, m(t)) .
$$

It is said that

$$
\left\|D_{\Delta}^{+} m(t)\right\|_{M} \leq\|G(t, m(t))\|_{M} .
$$

Because $\left\|V\left(t_{0}, X_{0}\right)\right\|_{M} \leq\left\|U_{0}\right\|_{M}$, we can obtain that

$$
\|V(t, X)\|_{M} \leq\|U(t)\|_{M}, \quad t \in \mathbb{T} .
$$

The proof is complete.

Now, we list some definitions about stability which will be used in the following discussion.

Definition 17. Let $h_{0}, h \in \Gamma, t \in \mathbb{T}$. Then one says that

(i) $h_{0}$ is finer than $h$ if there exists a $\rho>0$ and a function $\phi \in \mathscr{C} \mathscr{K}$ such that

$$
h_{0}(t, x)<\rho \text { implies } h(t, x) \leq \phi\left(t, h_{0}(t, x)\right) \text {; }
$$

(ii) $h_{0}$ is uniformly finer than $h$ if in (i) $\phi$ is independent of $t$.

Definition 18. Let $\lambda, A, B$ be positive constants $(\lambda<A, B<$ $A$ ). The system (18) is said to be

(i) practically stable if for any $0<\lambda<A$, the condition $\left\|X_{0}\right\|_{M}<\lambda$ implies $\|X(t)\|_{M}<A, t \geq t_{0}$ for $t_{0}, t \in \mathbb{T}$, where $X(t)=X\left(t, t_{0}, X_{0}\right)$ is any solution of (18);

(ii) practically quasi-stable if for any $\lambda, B, T>0$ and some $t_{0} \in \mathbb{T}$ with $t_{0}+T \in \mathbb{T}$, the condition $\left\|X_{0}\right\|_{M}<\lambda$ implies $\|X(t)\|_{M}<B, t \geq t_{0}+T, t \in \mathbb{T}$;

(iii) strongly practically stable if (i) and (ii) hold simultaneously;

(iv) practically asymptotically stable if (i) holds and for any $\epsilon>0$ there exists $T_{0}>0$ such that $t_{0}+T_{0} \in \mathbb{T}$ and $\left\|X_{0}\right\|<\lambda$ implies $\|X(t)\|<\epsilon, t \geq t_{0}+T_{0}$.

Definition 19. Let $\lambda, A, B$ be positive constants $(\lambda<A, B<$ $A), h, h_{0} \in \Gamma$. The system (18) is said to be

(PS1) $\left(h_{0}, h\right)$-practically stable if for any $0<\lambda<A$, the condition $h_{0}\left(t_{0}, X_{0}\right)<\lambda$ implies $h(t, X(t))<A, t \geq$ $t_{0}$, for some $t_{0}, t \in \mathbb{T}$, where $X(t)=X\left(t, t_{0}, X_{0}\right)$ is any solution of (18);

(PS2) $\left(h_{0}, h\right)$-practically quasi-stable if for any $\lambda, B, T>0$ and some $t_{0} \in \mathbb{T}$ with $t_{0}+T \in \mathbb{T}$, the condition $h_{0}\left(t_{0}, X_{0}\right)<\lambda$ implies $h(t, X(t))<B, t \geq t_{0}+T$, $t \in \mathbb{T}$

(PS3) $(h, h)$-strongly practically stable if (PS1) and (PS2) hold simultaneously;
(PS4) $\left(h_{0}, h\right)$-practically asymptotically stable if (PS1) holds and for any $\epsilon>0$ there exists $T_{0}>0$ such that $t_{0}+T_{0} \epsilon$ $\mathbb{T}$ and $h_{0}\left(t_{0}, X_{0}\right)<\lambda$ implies $h(t, X(t))<\epsilon, t \geq t_{0}+T_{0}$.

One can similarly define corresponding notion for the system (22).

Definition 20. Let $\lambda, A, B$ be positive constants $(\lambda<A, B<$ $A), Q_{0}, Q \in \Sigma$. Then we say that the system $(22)$ is $\left(Q_{0}, Q\right)$ practically stable if for any $0<\lambda<A$, the condition $Q_{0}$ $\left(\left\|U_{0}\right\|_{M}\right)<\lambda$ implies $Q\left(\|U(t)\|_{M}\right)<A, t \geq t_{0}, t \in \mathbb{T}$, where $U(t)=U\left(t, t_{0}, U_{0}\right)$ is any solution of $(22)$.

Other practical stability notions can be defined similarly.

\section{Theorem 21. Assume that}

$\left(A_{1}\right) \lambda$, $A$ are positive constants and $0<\lambda<A$;

$\left(A_{2}\right) h_{0}, h \in \Gamma, t \in \mathbb{T}, h_{0}$ is nondecreasing in $t$, and $h_{0}$ is uniformly finer than $h, h(t, X) \leq \phi\left(h_{0}(t, X)\right), \phi \in \mathscr{K}$, where $h_{0}(t, X)<\lambda$;

$\left(A_{3}\right)$ there exists $V \in C_{\mathrm{rd}}\left[\mathbb{T} \times K\left(\mathbb{R}^{n}\right)^{N}, \mathbb{R}^{N}\right]$, such that $V(t, X)$ is locally Lipschitzian in $X$ for each right-dense $t \in \mathbb{T}$ and for $a, b \in \mathscr{K}$. It holds that

$$
b(h(t, X)) \leq\|V(t, X)\|_{M} \leq a\left(h_{0}(t, X)\right) ;
$$

$$
\left(A_{4}\right) \text { for }(t, X) \in S(h, A) \text {, }
$$

$$
D_{\Delta}^{+} V(t, X) \leq G(t, V(t, X))
$$

where $G \in C_{\mathrm{rd}}\left[\mathbb{\square} \times \mathbb{R}^{N}, \mathbb{R}^{N}\right]$ is upper quasi-monotone nondecreasing in $U$ for each $t \in \mathbb{T}$ and $U(t)$ is the solution of (22);

$$
\left(A_{5}\right) \phi(\lambda)<A, a(\lambda)<b(A) .
$$

Then practical stability properties of system (22) imply the corresponding $\left(h_{0}, h\right)$-practical stability properties of (18).

Proof. Assume that (22) is practically stable; then for given $(a(\lambda), b(A))$, we have

$$
\left\|U_{0}\right\|_{M}<a(\lambda) \text { implying }\|U(t)\|_{M}<b(A), \quad t \geq t_{0} .
$$

Then by $\left(A_{2}\right)$ and $\left(A_{5}\right)$, it follows that $h\left(t_{0}, X_{0}\right) \leq \phi\left(h_{0}\left(t_{0}\right.\right.$, $\left.\left.X_{0}\right)\right)<\phi(\lambda)<A$. We claim that $h(t, X)<A$.

Indeed, if this were not true, there would exist a solution $X(t)$ of (18) with $h_{0}\left(t_{0}, X_{0}\right)<\lambda$ and $t_{1}>t_{0}, t_{1} \in \mathbb{T}$, such that $h\left(t_{1}, X\left(t_{1}\right)\right) \geq A, h(t, X(t))<A, t_{0} \leq t<t_{1}$. As $h_{0}\left(t_{0}, X_{0}\right)<$ $\lambda$, Theorem 16 together with $\left(A_{2}\right),\left(A_{3}\right)$ implies that

$$
\begin{gathered}
\|V(t, X)\|_{M}<\|U(t)\|_{M}, \quad t_{0} \leq t<t_{1}, \\
\left\|V\left(t_{0}, X_{0}\right)\right\|_{M} \leq a\left(h_{0}\left(t_{0}, X_{0}\right)\right)<a(\lambda), \\
b(A) \leq b\left(h\left(t_{1}, X\left(t_{1}, X\left(t_{1}\right)\right)\right)\right) \\
\leq\left\|V\left(t_{1}, X_{1}\right)\right\|_{M} \leq\left\|U\left(t_{1}\right)\right\|_{M} \leq b(A) .
\end{gathered}
$$

This contradiction proves that $h_{0}\left(t_{0}, X_{0}\right)<\lambda$ implies $h(t, X)$ $<A, t \geq t_{0}$. 
Next we prove that system $(18)$ is $\left(h_{0}, h\right)$-strongly practically stable. For given positive numbers $\lambda, A, B$, and $T$, suppose that (22) is strongly practically stable for positive numbers $a(\lambda), b(A), b(B)$, and $T$; this means we only need to prove $\left(h_{0}, h\right)$-practical quasi-stability of system (18). Practical quasi-stability of (22) means that $\left\|U_{0}\right\|_{M}<a(\lambda)$ implies $\|U(t)\|_{M}<b(B), t \geq t_{0}+T$ with $t_{0}+T \in \mathbb{T}$.

From the foregoing argument, since $\|V(t, X(t))\|_{M}<$ $a(\lambda)$, if $h_{0}\left(t_{0}, X_{0}\right)<\lambda$, we have $b(h(t, U)) \leq\|V(t, X)\|_{M} \leq$ $\|U(t)\|_{M}<b(B)$ for all $t \geq t_{0}+T$ if $h_{0}\left(t_{0}, X_{0}\right)<\lambda$; thus we have $h(t, X(t))<B, t \geq t_{0}+T$ provided $h_{0}\left(t_{0}, X_{0}\right)<\lambda$. Hence system $(18)$ is $\left(h_{0}, h\right)$-strongly practically stable.

Finally, we show that system $(18)$ is $\left(h_{0}, h\right)$-practically asymptotically stable. Now, let us suppose that (22) is practically asymptotically stable. This implies we only need to prove that for any given $\epsilon>0$, there exists $T_{0}>0$ with $t_{0}+T_{0} \in \mathbb{T}$ such that $t_{0}+T_{0} \geq T$ and $h_{0}\left(t_{0}, X_{0}\right)<\lambda$ implies $h(t, X(t))<B$, $t \geq t_{0}+T_{0}$ for system (18). Practical asymptotic stability of (22) means that

$$
\begin{array}{r}
\left\|U_{0}\right\|_{M}<a(\lambda) \text { implies }\left\|U\left(t, t_{0}, U_{0}\right)\right\|_{M}<b(B), \\
t \geq t_{0}+T_{0} .
\end{array}
$$

From the argument above, since $\left\|V\left(t_{0}, X_{0}\right)\right\|_{M}<a(\lambda)$ whenever $h_{0}\left(t_{0}, X_{0}\right)<\lambda$, we obtain

$$
b(h(t, X)) \leq\|V(t, X)\|_{M} \leq\|U(t, U)\|_{M}<b(B)
$$

for all $t \geq t_{0}+T_{0}$, if $h_{0}\left(t_{0}, X_{0}\right)<\lambda$. Thus we have $h(t, X)<B$, $t \geq t_{0}+T_{0}$, provided $h_{0}\left(t_{0}, X_{0}\right)<\lambda$. Hence system (18) is $\left(h_{0}, h\right)$-strongly practically stable.

The proof is complete.

Theorem 22. Suppose that the conditions of Theorem 21 are satisfied except that condition $\left(A_{3}\right)$ is replaced by

$$
\begin{array}{r}
\left(A_{6}\right) Q_{0}, Q \in \sum \text { and for } a, b \in \mathscr{K} \\
Q\left(\|V(t, X)\|_{M}\right) \geq b(h(t, X)), \\
Q_{0}\left(\|V(t, X)\|_{M}\right) \leq a\left(h_{0}(t, X)\right) .
\end{array}
$$

Then $\left(Q_{0}, Q\right)$-practical stability properties of system (22) imply the corresponding $\left(h_{0}, h\right)$-practical stability properties of the system (18).

Proof. Assume that $(22)$ is $\left(Q_{0}, Q\right)$-practically stable. Then we have $(a(\lambda), b(A))$, such that

$$
\begin{array}{r}
Q_{0}\left(\left\|U_{0}\right\|_{M}\right)<a(\lambda) \text { implies } Q\left(\left\|U\left(t, t_{0}, U_{0}\right)\right\|_{M}\right)<b(A), \\
t \geq t_{0} .
\end{array}
$$

Suppose that the $\left(h_{0}, h\right)$-practical stability of (18) does not hold; then, arguing as in Theorem 16 , by $\left(A_{2}\right),\left(A_{6}\right)$, we can show that

$$
\begin{aligned}
Q_{0}(\| & \left.V\left(t, X\left(t, t_{0}, X_{0}\right)\right) \|_{M}\right) \\
& \leq a\left(h_{0}\left(t, X\left(t, t_{0}, X_{0}\right)\right)\right) \\
& \leq a\left(h_{0}\left(t_{1}, X\left(t_{1}, t_{0}, X_{0}\right)\right)\right) \leq a(\lambda) .
\end{aligned}
$$

Then using $\left(A_{6}\right)$, we have

$$
\begin{aligned}
b(A) & \leq b\left(h\left(t_{1}, X\left(t_{1}, t_{0}, X_{0}\right)\right)\right) \\
& \leq Q\left(\left\|V\left(t_{1}, X\left(t_{1}, t_{0}, X_{0}\right)\right)\right\|_{M}\right) \\
& \leq Q\left(\left\|U\left(t_{1}, U\left(t_{1}, t_{0}, U_{0}\right)\right)\right\|_{M}\right) \\
& <b(A),
\end{aligned}
$$

which is a contradiction. The proof is complete.

\section{Conflict of Interests}

The authors declare that there is no conflict of interests regarding the publication of this paper.

\section{Authors' Contribution}

All authors completed the paper together. All authors read and approved the final paper.

\section{Acknowledgments}

The authors would like to thank the reviewers for their valuable suggestions and comments. This paper is supported by the National Natural Science Foundation of China (11271106) and the Natural Science Foundation of Hebei Province of China (A2013201232).

\section{References}

[1] J. P. Lasalle and S. Lefschetz, Stability by Lyapunov's Direct Method with Applications, Academic Press, New York, NY, USA, 1961.

[2] N. Rouche, P. Habets, and M. Laloy, Stability Theory by Lyapunov's Direct Method, Springer, New York, NY, USA, 1997.

[3] V. Lakshmikantham and X. Z. Liu, Stability Analysis in Terms of Two Measures, World Scientific, Singapore, 1993.

[4] S. M. S. de Godoy and M. A. Bená, "Stability criteria in terms of two measures for functional differential equations," Applied Mathematics Letters, vol. 18, no. 6, pp. 701-706, 2005.

[5] P. Wang and H. Lian, "On the stability in terms of two measures for perturbed impulsive integro-differential equations," Journal of Mathematical Analysis and Applications, vol. 313, no. 2, pp. 642-653, 2006.

[6] P. Wang and Z. Zhan, "Stability in terms of two measures of dynamic system on time scales," Computers and Mathematics with Applications, vol. 62, no. 12, pp. 4717-4725, 2011. 
[7] V. Lakshmikantham, S. Leela, and A. A. Martynyuk, Practical Stability of Nonlinear Systems, World Scientific, Singapore, 1990.

[8] Y. Zhang and J. Sun, "Practical stability of impulsive functional differential equations in terms of two measurements," Computers and Mathematics with Applications, vol. 48, no. 10-11, pp. 1549-1556, 2004

[9] P. Wang and X. Liu, "Practical stability of impulsive hybrid differential systems in terms of two measures on time scales," Nonlinear Analysis: Theory, Methods and Applications, vol. 65, no. 11, pp. 2035-2042, 2006.

[10] P. Wang, M. Wu, and Y. Wu, "Practical stability in terms of two measures for discrete hybrid systems," Nonlinear Analysis: Hybrid Systems, vol. 2, no. 1, pp. 58-64, 2008.

[11] S. Sun, Z. Han, E. Akin-Bohner, and P. Zhao, "Practical stability in terms of two measures for hybrid dynamic systems," Bulletin of the Polish Academy of Sciences. Mathematics, vol. 58, no. 3, pp. 221-237, 2010.

[12] S. G. Hristova and A. Georgieva, "Pratical Stability in terem of two measures for impulsive differential equations with "Supremum',' International Journal of Differential Equations, vol. 2011, Article ID 703189, 13 pages, 2011.

[13] T. G. Bhaskar, V. Lakshmikantham, and J. V. Devi, Set Differential Equations in a Metric Space, Cambridge Scientific, Cambridge, UK, 2005.

[14] A. J. B. Lopes Pinto, F. S. De Blasi, and F. Iervolino, "Uniqueness and existence theorems for differential equations with compact convex valued solutions," Bollettino della Unione Matematica Italiana, vol. 3, pp. 47-54, 1970.

[15] V. Lakshmikantham and A. S. Vatsala, "Set differential equations and monotone flows," Nonlinear Dynamics and Systems Theory, vol. 3, no. 2, pp. 33-43, 2003.

[16] V. Lakshmikantham, S. Leela, and A. S. Vatsala, "Set-valued hybrid differential equations and stability in terms of two measures," Journal of Hybrid Systems, vol. 2, no. 2, pp. 169-188, 2002.

[17] T. G. Bhaskar and J. V. Devi, "Stability criteria for set differential equations," Mathematical and Computer Modelling, vol. 41, no. 11-12, pp. 1371-1378, 2005.

[18] T. G. Bhaskar and J. V. Devi, "Nonuniform stability and boundedness criteria for set differential equations," Applicable Analysis, vol. 84, no. 2, pp. 131-143, 2005.

[19] B. Ahmad and S. Sivasundaram, "Setvalued perturbed hybrid integro-differential equations and stability in terms of two measures," Dynamic Systems and Applications, vol. 16, no. 2, pp. 299-310, 2007.

[20] N. D. Phu, L. T. Quang, and T. T. Tung, "Stability criteria for set control differential equations," Nonlinear Analysis: Theory, Methods and Applications, vol. 69, no. 11, pp. 3715-3721, 2008.

[21] N. N. Tu and T. T. Tung, "Stability of set differential equations and applications," Nonlinear Analysis: Theory, Methods and Applications, vol. 71, no. 5-6, pp. 1526-1533, 2009.

[22] W. T. Girton, Set-valued differential equations on time scales [Ph.D. thesis], Florida Institute of Technology, Melbourne, Fla, USA, 2006.

[23] B. Ahmad and S. Sivasundaram, "Basic results and stability criteria for set valued differential equations on time scales," Communications in Applied Analysis, vol. 11, no. 3-4, pp. 419428, 2007.
[24] S. Hong, "Differentiability of multivalued functions on time scales and applications to multivalued dynamic equations," Nonlinear Analysis: Theory, Methods and Applications, vol. 71, no. 9, pp. 3622-3637, 2009.

[25] S. Hong, "Stability criteria for set dynamic equations on time scales," Computers and Mathematics with Applications, vol. 59, no. 11, pp. 3444-3457, 2010. 


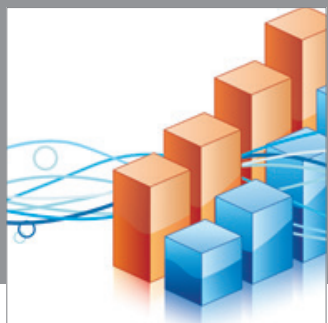

Advances in

Operations Research

mansans

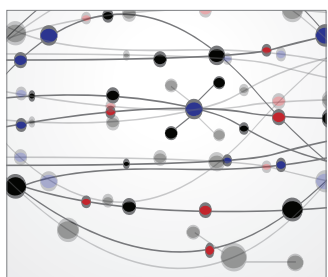

The Scientific World Journal
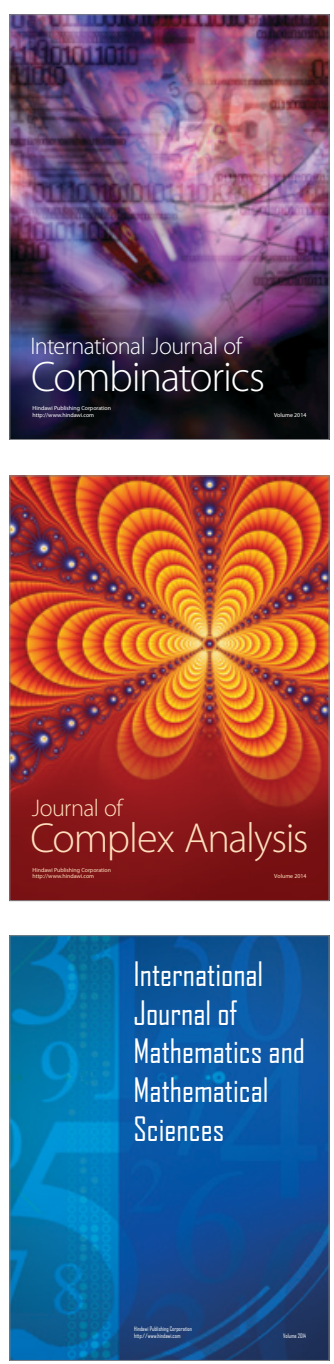
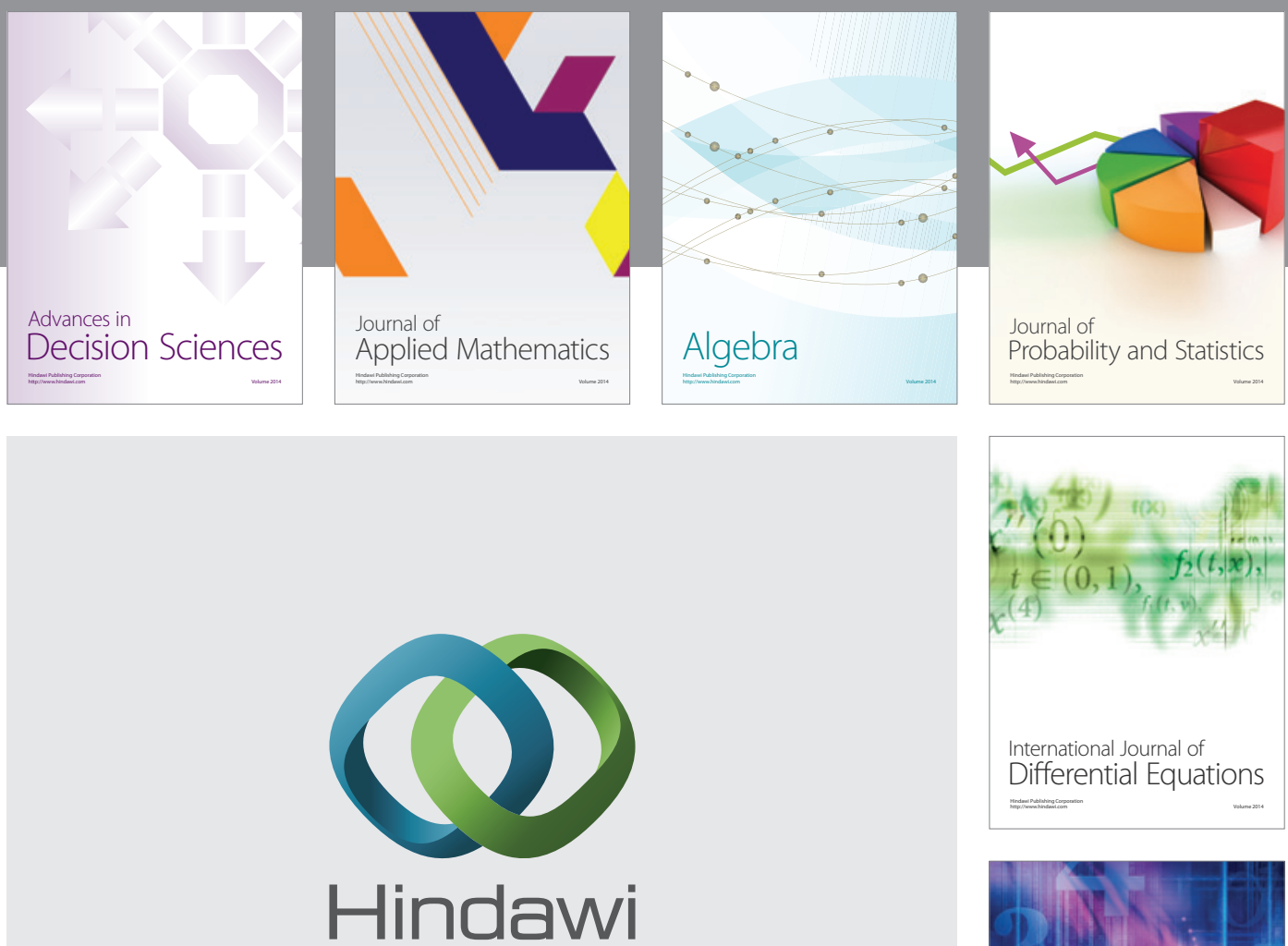

Submit your manuscripts at http://www.hindawi.com
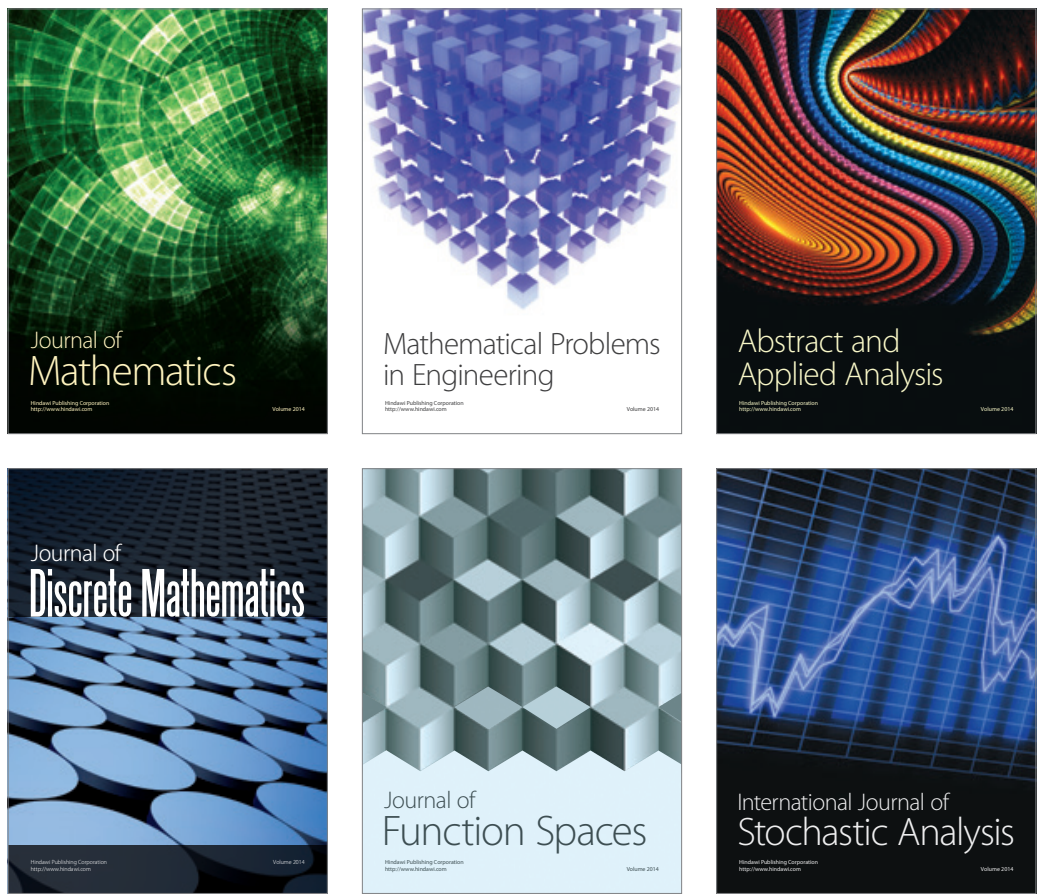

Journal of

Function Spaces

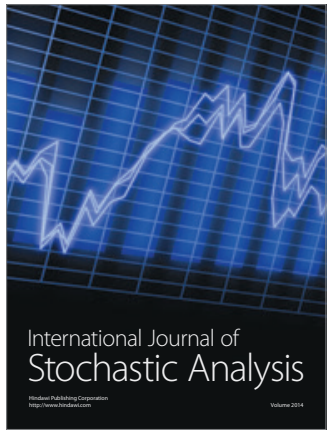

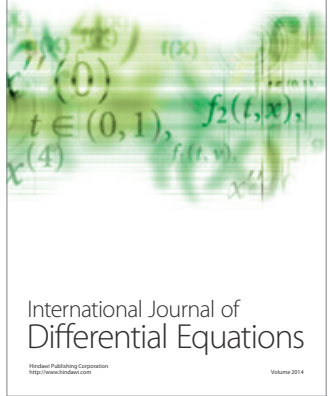
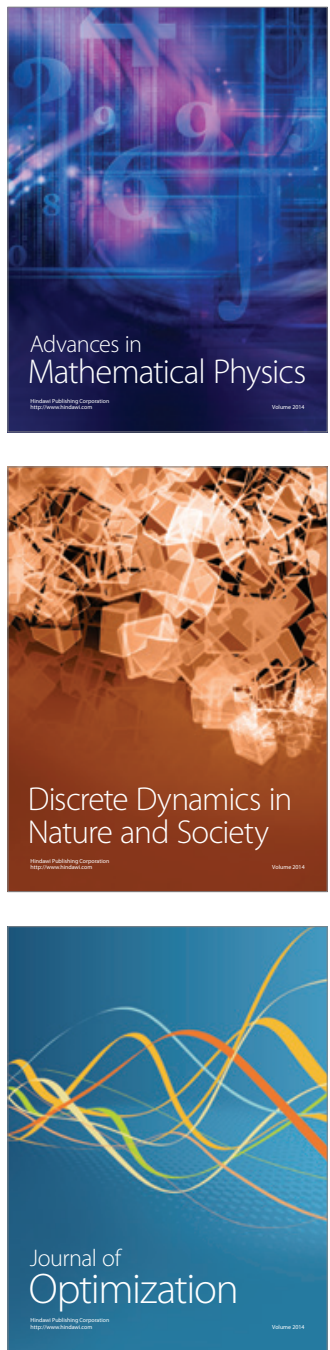